Braz J Med Biol Res, January 2012, Volume 45(1) 68-71

doi: 10.1590/S0100-879X2011007500165

Serum levels of brain-derived neurotrophic factor correlate with the number of T2 MRI lesions in multiple sclerosis

\author{
E.R. Comini-Frota, D.H. Rodrigues, E.C. Miranda, D.G. Brum, D.R. Kaimen-Maciel, E.A. Donadi \\ and A.L. Teixeira
}

The Brazilian Journal of Medical and Biological Research is partially financed by

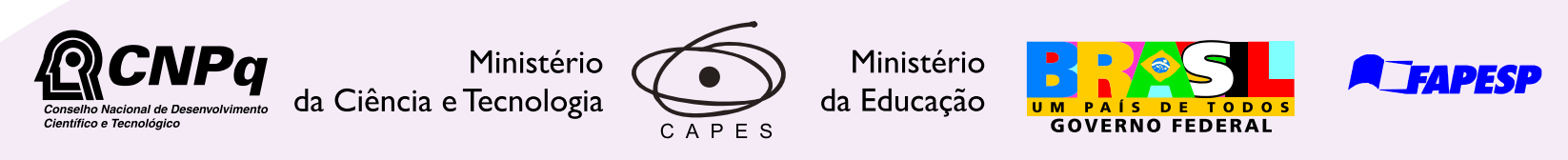
Institutional Sponsors
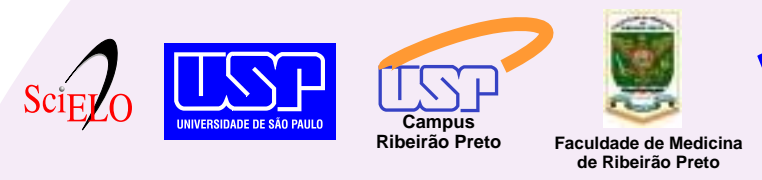

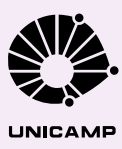

SHIMADZu

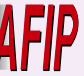

Associaçä Fundo
delmentivo
âesquisa

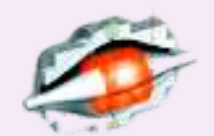




\title{
Serum levels of brain-derived neurotrophic factor correlate with the number of T2 MRI lesions in multiple sclerosis
}

\author{
E.R. Comini-Frota ${ }^{1}$, D.H. Rodrigues ${ }^{2}$, E.C. Miranda ${ }^{3}$, D.G. Brum ${ }^{4}$, \\ D.R. Kaimen-Maciel ${ }^{5}$, E.A. Donadi ${ }^{4}$ and A.L. Teixeira ${ }^{1,2}$ \\ ${ }^{1}$ Unidade de Neurologia, Hospital Universitário, Universidade Federal de Minas Gerais, Belo Horizonte, MG, Brasil \\ 2Laboratório de Imunofarmacologia, Instituto de Ciências Biológicas, \\ Universidade Federal de Minas Gerais, Belo Horizonte, MG, Brasil \\ ${ }^{3}$ Ecoar Diagnostic Center, Belo Horizonte, MG, Brasil \\ ${ }^{4}$ Hospital das Clínicas, Faculdade de Medicina de Ribeirão Preto, \\ Universidade de São Paulo, Ribeirão Preto, SP, Brasil \\ 5 Unidade de Neurologia, Hospital Universitário, Universidade Estadual de Londrina, Londrina, PR, Brasil
}

\begin{abstract}
The objective of the present study was to determine if there is a relationship between serum levels of brain-derived neurotrophic factor (BDNF) and the number of T2/fluid-attenuated inversion recovery (T2/FLAIR) lesions in multiple sclerosis (MS). The use of magnetic resonance imaging (MRI) has revolutionized the study of MS. However, MRI has limitations and the use of other biomarkers such as BDNF may be useful for the clinical assessment and the study of the disease. Serum was obtained from 28 MS patients, 18-50 years old (median 38), 21 women, 0.5-10 years (median 5) of disease duration, EDSS 1-4 (median 1.5) and 28 healthy controls, 19-49 years old (median 33), 19 women. BDNF levels were measured by ELISA. T1, T2/FLAIR and gadolinium-enhanced lesions were measured by a trained radiologist. BDNF was reduced in MS patients (median [range] pg/mL; 1160 [352.6-2640]) compared to healthy controls (1640 [632.4-4268]; $P=0.03$, Mann-Whitney test) and was negatively correlated (Spearman correlation test, $r=-0.41 ; P=0.02)$ with T2/FLAIR (11-81 lesions, median 42). We found that serum BDNF levels were inversely correlated with the number of T2/FLAIR lesions in patients with MS. BDNF may be a promising biomarker of MS.
\end{abstract}

Key words: Multiple sclerosis; Biomarkers; Neurotrophins; BDNF; Neuroimaging

\section{Introduction}

Multiple sclerosis (MS) is the most prevalent demyelinating disease affecting young people around the world. The use of magnetic resonance imaging (MRI) in clinical practice revolutionized the process of MS diagnosis. MRI is also important for the clinical follow-up of MS patients, contributing to the process of definition of MS relapses and disease progression. Indeed, the burden of T1 and T2 lesions has been reported to correlate with MS progression $(1,2)$. As a result, MRI is being increasingly used as a marker for the diagnosis and follow-up of disease activity and progression in MS patients $(3,4)$.

Besides imaging parameters, treatment decisions may benefit from other accessible clinical biomarkers. Some putative biomarkers have been proposed for MS (5). However, at present, no validated biomarkers are available to diagnose the disease, to monitor or predict its progression, or to aid in the assessment of the effects of early treatment (6).

Brain-derived neurotrophic factor (BDNF) belongs to a class of proteins expressed in the nervous system of mammals called neurotrophins, which have been related to neuronal development and survival (7). BDNF levels are altered in a variety of neurological diseases. For instance, serum BDNF from Alzheimer's disease patients is decreased when compared to healthy controls (8). BDNF is also diminished in patients with Parkinson's disease and correlates with the severity of motor symptoms and the span of this disease (9). Increased expression of a high-affinity BDNF receptor was found in T-cell lines from MS patients (10), whereas low BDNF synthesis by peripheral blood mononuclear cells (PBMC) was related to reduced neuroprotection $(11,12)$.

Correspondence: E.R. Comini-Frota, Departamento de Neurologia, Hospital das Clínicas da UFMG, Av. Alfredo Balena, 110, 30100-130 Belo Horizonte, MG, Brasil. E-mail: elizcomini@gmail.com.br

Received July 14, 2011. Accepted November 21, 2011. Available online November 23, 2011. Published January 16, 2012. 
Therefore, BDNF may be a potential clinical marker of disease progression.

In the present study, we determined if there was a correlation between the number of MRI T1, T2, and gadolinium-enhanced $\left(\mathrm{Gad}^{+}\right)$lesions in patients with relapsing remittent MS (RRMS) and BDNF serum levels.

\section{Patients and Methods}

Patients with a definite diagnosis of RRMS according to the McDonald criteria (13) were selected for this study if they met the following inclusion criteria: less than 10 years of disease, clinical and/or radiological evidence of at least one relapse in the last 12 months, and Expanded Disability Status Scale (EDSS) score below 5 (14). Relapses were defined as an acute new symptom or worsening of old symptoms topographically related to a white matter lesion in the central nervous system (CNS) that lasted over $24 \mathrm{~h}$ (14). Blood was collected at least 3 months after the last relapse. For comparison, healthy subjects were invited to participate. Patients and controls were submitted to one MRI scan.

MRI was performed within 1 week after collecting the blood sample using a 1.5 Tesla apparatus (GE Xcite II, 2004, USA). T2-weighted images, conventional spin-echo T1-weighted images, and fluid-attenuated inversion recovery (FLAIR) images were obtained in sagittal, coronal and axial sequences (15). A radiologist blind to the medical status of the patients analyzed all exams and performed manual counting of the number of T1 hypointense, T2/ FLAIR hyperintense and $\mathrm{Gad}^{+}$lesions in axial images with 3-mm long slices.

These data were correlated with the serum levels of BDNF assessed by ELISA. All blood samples were collected in the morning, and the serum was separated and frozen at $-70^{\circ} \mathrm{C}$ until the time for BDNF measurement. BDNF was measured by sandwich ELISA according to the protocol provided by the manufacturer (R\&D Systems, USA). Assays were made in duplicate on the same day and intra-assay variability was less than $5 \%$.

Nonparametric analysis was performed using MannWhitney and Kruskal-Wallis tests for two or more unpaired data, Wilcoxon and Friedman tests for two or more paired data, and the Spearman test for correlation. The $P$ value was set at 0.05 .

\section{Results}

The demographic and clinical features of MS patients and controls are summarized in Table 1.

BDNF was reduced in MS patients (median [range] pg/ $\mathrm{mL} ; 1160$ [352.6-2640]) compared to healthy controls (1640 [632.4-4268]; $P=0.03$, Mann-Whitney test). No correlation

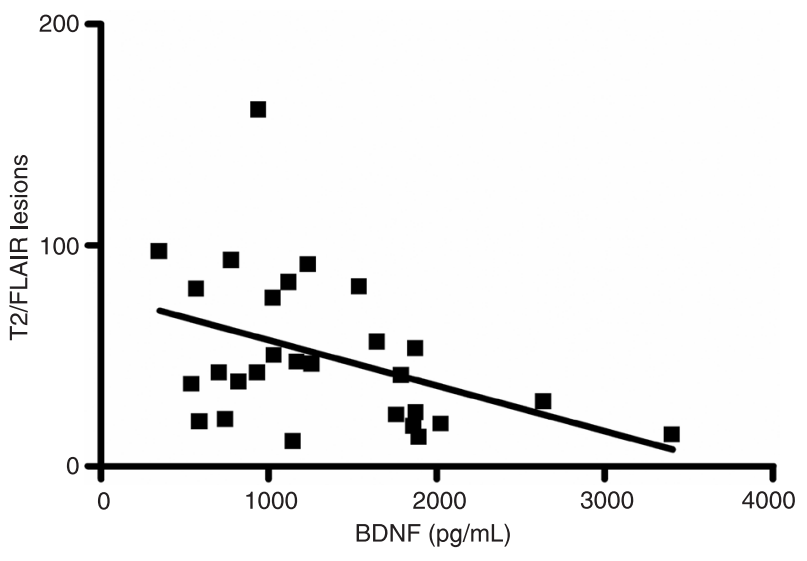

Figure 1. BDNF levels are inversely correlated with the number of T2/FLAIR lesions determined by MRI. Serum from MS patients $(N=28)$ was collected and BDNF levels were measured by ELISA. There was a significant negative correlation $(P=0.0292)$ between BDNF levels and T2/FLAIR lesions. BDNF = brain-derived neurotrophic factor; T2/FLAIR = T2/fluid-attenuated inversion recovery.

was found between BDNF levels and age $(P=0.1)$ or gender $(P=0.1)$ of patients and controls, or between BDNF levels and time of disease onset $(P=0.2)$ or EDSS $(P=0.2)$.

BDNF levels in serum from patients correlated negatively with the number of T2/FLAIR lesions $(r=-0.41 ; P=0.02$; Figure 1). No correlation was found between BDNF levels and T1 $(P=0.46)$ or $\mathrm{Gad}^{+}$lesions $(P=0.85)$.

\section{Discussion}

We report here that serum levels of BDNF are inversely correlated with the number of T2/FLAIR hyperintense lesions in MS patients.

Previous reports have established a correlation between 
the burden of T2 lesions and clinical outcome during shortterm follow-up $(16,17)$. Furthermore, the load of T2 lesions above all other MRI findings was the best marker of MS activity in the early years of the disease and the best parameter correlated with disability in long-term follow-up (4). Therefore, BDNF may be seen as a promising biomarker of MS.

Since circulating levels of BDNF are correlated with its CNS concentration (18) and that load of T2/FLAIR lesions indicates demyelination of various degrees and neuronal damage $(19,20)$, decreased circulating levels of BDNF may be associated with reduced synthesis of the molecule in the CNS and, hence, loss of neuroprotective support. Indeed, the present result agrees with the putative protective role of BDNF in neurodegenerative diseases $(21,22)$. MS patients present lower levels of circulating BDNF compared to healthy controls, but these levels tend to increase after a relapse $(23,24)$. An inverse correlation between BDNF synthesis by PBMC and cognitive performance of MS patients has also been reported, reinforcing the view of BDNF as a neuroprotective molecule (25). Moreover, immune cells can secrete BDNF, and a positive association between synthesis of BDNF by PBMC and white matter volume has been reported to occur in MS (26). Some investigators even hypothesized that neuronal repair mechanisms act

\section{References}

1. Polman $\mathrm{CH}$, Wolinsky JS, Reingold SC. Multiple sclerosis diagnostic criteria: three years later. Mult Scler 2005; 11: 5-12.

2. Rashid W, Miller DH. Recent advances in neuroimaging of multiple sclerosis. Semin Neurol 2008; 28: 46-55.

3. Amato MP, Portaccio E, Goretti B, Zipoli V, Battaglini M, Bartolozzi ML, et al. Association of neocortical volume changes with cognitive deterioration in relapsing-remitting multiple sclerosis. Arch Neurol 2007; 64: 1157-1161.

4. Rudick RA, Lee JC, Simon J, Fisher E. Significance of T2 lesions in multiple sclerosis: A 13-year longitudinal study. Ann Neurol 2006; 60: 236-242.

5. Audoin B, Guye M, Reuter F, Au Duong MV, Confort-Gouny $S$, Malikova I, et al. Structure of WM bundles constituting the working memory system in early multiple sclerosis: a quantitative DTI tractography study. Neuroimage 2007; 36 : 1324-1330.

6. Teunissen CE, Dijkstra C, Polman C. Biological markers in CSF and blood for axonal degeneration in multiple sclerosis. Lancet Neurol 2005; 4: 32-41.

7. Ottervald J, Franzen B, Nilsson K, Andersson LI, Khademi $M$, Eriksson B, et al. Multiple sclerosis: Identification and clinical evaluation of novel CSF biomarkers. J Proteomics 2010; 73: 1117-1132.

8. Reichardt LF. Neurotrophin-regulated signalling pathways. Philos Trans R Soc Lond B Biol Sci 2006; 361: 1545-1564.

9. Laske C, Stransky E, Leyhe T, Eschweiler GW, Maetzler W, Wittorf A, et al. BDNF serum and CSF concentrations in Alzheimer's disease, normal pressure hydrocephalus and healthy controls. J Psychiatr Res 2007; 41: 387-394. up to a threshold above which the repair cannot continue, and this threshold is correlated with the accumulation of lesions on T2 $(4,17)$.

The absence of correlation between T1 lesions and BDNF levels may be due to the T1 lesions themselves indicating chronic lesions (19). If BDNF is considered to be part of a repair mechanism, it cannot be correlated with lesions that express a chronic neuronal loss. Moreover, T1 and $\mathrm{Gad}^{+} \mathrm{le}-$ sions may disappear after some time, but every lesion, new or old, leaves a footprint in T2/FLAIR imaging (20).

Our study has some drawbacks such as the small number of MS patients enrolled and the fact that it was not possible to stratify the sample and to control the effect of immunomodulatory treatment on BDNF levels. Manual counting of MRI lesions is not the most accurate form to measure the burden of demyelinating lesions, but is the most feasible one in clinical practice.

In conclusion, BDNF may be used as a biomarker of MS, although further studies are needed to confirm this preliminary finding.

\section{Acknowledgments}

\author{
Research supported by CNPq and FAPEMIG.
}

10. Scalzo P, Kummer A, Bretas TL, Cardoso F, Teixeira AL. Serum levels of brain-derived neurotrophic factor correlate with motor impairment in Parkinson's disease. J Neurol 2010; 257: 540-545.

11. De Santi L, Cantalupo L, Tassi M, Raspadori D, Cioni C, Annunziata P. Higher expression of BDNF receptor gp145trkB is associated with lower apoptosis intensity in T cell lines in multiple sclerosis. J Neurol Sci 2009; 277: 65-70.

12. Azoulay D, Urshansky N, Karni A. Low and dysregulated BDNF secretion from immune cells of MS patients is related to reduced neuroprotection. J Neuroimmunol 2008; 195: 186-193.

13. McDonald WI, Compston A, Edan G, Goodkin D, Hartung HP, Lublin FD, et al. Recommended diagnostic criteria for multiple sclerosis: guidelines from the International Panel on the diagnosis of multiple sclerosis. Ann Neurol 2001; 50: 121-127.

14. Linker RA, Lee DH, Demir S, Wiese S, Kruse N, Siglienti I, et al. Functional role of brain-derived neurotrophic factor in neuroprotective autoimmunity: therapeutic implications in a model of multiple sclerosis. Brain 2010; 133: 2248-2263.

15. Kurtzke JF. Rating neurologic impairment in multiple sclerosis: an expanded disability status scale (EDSS). Neurology 1983; 33: 1444-1452.

16. Filippi M, Paty DW, Kappos L, Barkhof F, Compston DA, Thompson AJ, et al. Correlations between changes in disability and T2-weighted brain MRI activity in multiple sclerosis: a follow-up study. Neurology 1995; 45: 255-260.

17. Barkhof $F$. The clinico-radiological paradox in multiple sclerosis revisited. Curr Opin Neurol 2002; 15: 239-245. 
18. Teixeira AL, Barbosa IG, Diniz BS, Kummer A. Circulating levels of brain-derived neurotrophic factor: correlation with mood, cognition and motor function. Biomark Med 2010; 4: 871-887.

19. Zivadinov R, Stosic M, Cox JL, Ramasamy DP, Dwyer MG. The place of conventional MRI and newly emerging MRI techniques in monitoring different aspects of treatment outcome. J Neurol 2008; 255 (Suppl 1): 61-74.

20. Simon JH. MRI in multiple sclerosis. Phys Med Rehabil Clin N Am 2005; 16: 383-409, viii.

21. Nagahara AH, Merrill DA, Coppola G, Tsukada S, Schroeder BE, Shaked GM, et al. Neuroprotective effects of brainderived neurotrophic factor in rodent and primate models of Alzheimer's disease. Nat Med 2009; 15: 331-337.

22. Zuccato C, Ciammola A, Rigamonti D, Leavitt BR, Goffredo $\mathrm{D}$, Conti $\mathrm{L}$, et al. Loss of huntingtin-mediated BDNF gene transcription in Huntington's disease. Science 2001; 293:
493-498.

23. Sarchielli P, Greco L, Stipa A, Floridi A, Gallai V. Brain-derived neurotrophic factor in patients with multiple sclerosis. J Neuroimmunol 2002; 132: 180-188.

24. Frota ER, Rodrigues DH, Donadi EA, Brum DG, Maciel $D R$, Teixeira AL. Increased plasma levels of brain derived neurotrophic factor (BDNF) after multiple sclerosis relapse. Neurosci Lett 2009; 460: 130-132.

25. Patanella AK, Zinno M, Quaranta D, Nociti V, Frisullo G, Gainotti $G$, et al. Correlations between peripheral blood mononuclear cell production of BDNF, TNF-alpha, IL-6, IL10 and cognitive performances in multiple sclerosis patients. J Neurosci Res 2010; 88: 1106-1112.

26. Weinstock-Guttman B, Zivadinov R, Tamano-Blanco M, Abdelrahman N, Badgett D, Durfee J, et al. Immune cell BDNF secretion is associated with white matter volume in multiple sclerosis. J Neuroimmunol 2007; 188: 167-174. 